\title{
Leveraging Higher Order Dependencies between Features for Text Classification
}

\author{
Murat C. Ganiz ${ }^{1,3}$, Nikita I. Lytkin ${ }^{2}$, and William M. Pottenger ${ }^{2,3}$ \\ 1 Department of Computer Science \\ Lehigh University, USA \\ 2 Department of Computer Science \\ Rutgers, The State University of New Jersey, USA \\ 3 DIMACS \\ Rutgers, The State University of New Jersey, USA
}

\begin{abstract}
Traditional machine learning methods only consider relationships between feature values within individual data instances while disregarding the dependencies that link features across instances. In this work, we develop a general approach to supervised learning by leveraging higher-order dependencies between features. We introduce a novel Bayesian framework for classification named Higher Order Naive Bayes (HONB). Unlike approaches that assume data instances are independent, HONB leverages co-occurrence relations between feature values across different instances. Additionally, we generalize our framework by developing a novel data-driven space transformation that allows any classifier operating in vector spaces to take advantage of these higher-order cooccurrence relations. Results obtained on several benchmark text corpora demonstrate that higher-order approaches achieve significant improvements in classification accuracy over the baseline (first-order) methods.
\end{abstract}

Keywords: machine learning, text classification, higher order learning, statistical relational learning, higher order naive bayes, higher order support vector machine.

\section{Introduction}

A well known problem in real-world applications of machine learning methods is that expert labeling of large amounts of data for training a classifier is prohibitively expensive. Often in practice, only a small amount of labeled data is available for training. Traditional methods of classification treat individual data instances independently. In this case, however, a small training set makes an adequate estimation of the model parameters of a classifier very challenging. Prior work has demonstrated the value of leveraging explicit 1223 as well as implicit 4.5] link information within data in order to provide a richer data representation for model estimation.

In Sect. 4.1, we build on a graph-based data representation from our prior work [4] and introduce a novel Bayesian framework for classification named Higher Order

W. Buntine et al. (Eds.): ECML PKDD 2009, Part I, LNAI 5781, pp. 375-390, 2009.

(C) Springer-Verlag Berlin Heidelberg 2009 
Naive Bayes (HONB). Unlike approaches that assume data instances are independent, HONB leverages co-occurrence relations between feature values across different instances. We term these implicit co-occurrence relations higher-order paths. Features (e.g., words in documents of a text collection) are richly connected by such higher-order paths, and a model built by HONB exploits this rich connectivity.

We further generalize our framework in Sect. 4.2 by developing a novel datadriven space transformation that allows any classifier operating in vector spaces to take advantage of relational dependencies captured by higher-order paths between features.

We evaluate the proposed methods 1 on several benchmark text corpora across a wide range of training set sizes in Sect. 5 . In that section, we also draw comparisons with the results reported in [6], where a word clustering approach was proposed for dealing with small and sparse training data for text classification.

The paper is organized as follows. Related work is discussed in Sect. 2. In Sect. 3. we provide the necessary background for development of the proposed methods described in Sect. 4. Experimental results are presented in Sect. 5. A discussion of the effects of leveraging higher-order dependencies for text classification is provided in Sect. 6. Concluding remarks are made in Sect. 7 .

\section{Related Work}

Our motivation for using higher-order dependencies for classification stems from advances in the areas of link mining [7] and information retrieval. In addition to (or sometimes instead of) using the more traditional data representation by feature vectors characterizing each data instance independently of the others, link-based approaches 38 899 to collective classification leverage explicit dependencies, or links, within networked data [9. Several studies [12/3] have shown that collective classification can achieve significant reductions in classification errors by performing inferences about multiple data instances simultaneously. However, such methods are context-dependent and are therefore not designed to classify single data instances. This restriction limits the domain of applicability of link-based classifiers.

In this work, we propose classification methods that leverage higher-order dependencies in the form of implicit links between instances and features of the training data. Unlike collective classifiers, methods presented in this work maintain the ability to classify single data instances without requiring any additional context information.

In [5], we gave a mathematical proof supported by empirical results of the dependence of Latent Semantic Indexing (LSI) [10], a technique often used in text mining and information retrieval, on higher-order relations and in particular on higherorder term co-occurrences expressed via higher-order paths. A higher-order path is exemplified in Fig. 10(reproduced from [5]) in the context of text data. Figure 1 depicts three documents, D1, D2 and D3, each containing two terms represented by

1 "Systems and Methods for Data Transformation for Supervised Machine Learning," M.C. Ganiz, N.I. Lytkin and W.M. Pottenger, U.S. Patent Pending 61/185255. 
the letters A, B, C and D. Shown below the three documents in Fig. 1 is a higherorder path that links term A with term D through B and C. This path contains three edges and is therefore referred to as a third-order path.

Higher-order relations play an im-

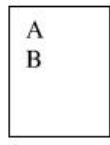

D1

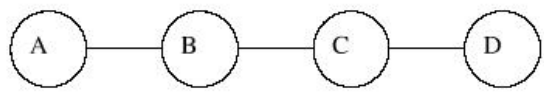

$1^{\text {st }}$ order term co-occurrence $\{A, B\},\{B, C\},\{C, D\}$

$2^{\text {nd }}$ order term co-occurrence $\{A, C\},\{B, D\}$

$3^{\text {rd }}$ order term co-occurrence $\{A, D\}$

Fig. 1. Higher-order co-occurrences [5] portant role in many other systems for text mining and information retrieval. In [11, higher-order associations were used for rule mining in textual data. Higher-order co-occurrences were used in 12 for solving a component of the problem of lexical choice, which identifies synonyms in a given context. In another effort, 13. used second-order co-occurrences for improving the runtime performance of LSI. Higher-order co-occurrences have also been used in other applications including word sense disambiguation 14 and stemming [15].

It should be noted that our framework extends beyond the textual domain. In other words, instances D1, D2 and D3 from Fig. 1 need not be text documents - they may be records in a database, or instances in a labeled training dataset. Likewise, features A, B, C, etc. need not be terms - they may be values in a database record, or feature-value pairs in a data instance.

In fact, in [4 we have successfully used higher-order paths for capturing dependencies amongst data instances when modeling time series data for anomaly detection in the Border Gateway Protocol, which constitutes the backbone of the Internet's routing infrastructure. The approach presented in this work builds on the data representation introduced in our prior work [4 and also described in Sect. 3.2

\section{Background}

In this section we review parts of the Bayesian learning theory (Sect. 3.1) and the data representation (Sect. 3.2) underlying the development of approaches presented in Sect. 4. Although the methods discussed in this work are not limited to a particular application domain, here we restrict our attention to textual data. We assume the input space to be an $n$-dimensional binary vector space where each document is represented by a vector whose non-zero coordinates correspond to terms present in the document.

Formally, let $W=\left\{w_{1}, \ldots, w_{n}\right\}$ denote the set of binary features that correspond to terms in the vocabulary. Any document $d$ can therefore be represented by an $n$-dimensional binary vector $w(d)=\left(w_{1}(d), \ldots, w_{n}(d)\right)$, where

$$
w_{i}(d)=\left\{\begin{array}{l}
1, \text { if document } d \text { contains term } w_{i} \\
0, \text { otherwise. }
\end{array}\right.
$$


For convenience of further exposition, we will simply write $d$ to denote the corresponding vector $w(d)$.

\subsection{Bayesian Learning Theory}

Given a document $d$ and two classes $c_{1}$ and $c_{2}$, the Bayes discriminant function can be written as

$$
f(d)=\log \frac{P\left(c_{1} \mid d\right)}{P\left(c_{2} \mid d\right)}=\log \frac{P\left(d \mid c_{1}\right) P\left(c_{1}\right)}{P\left(d \mid c_{2}\right) P\left(c_{2}\right)},
$$

where

$$
P\left(d \mid c_{j}\right)=P\left(w_{1}(d), \ldots, w_{n}(d) \mid c_{j}\right), \forall j \in\{1,2\},
$$

is the conditional likelihood of document $d$ belonging to class $c_{j}$, and $P\left(c_{j}\right)$ is the prior probability of class $c_{j}$.

By assumption of mutual independence of terms given a class, the conditional likelihood (2) becomes

$$
P\left(w_{1}(d), \ldots, w_{n}(d) \mid c_{j}\right)=\prod_{i=1}^{n} P\left(w_{i}(d) \mid c_{j}\right),
$$

where $P\left(w_{i}(\cdot) \mid c_{j}\right)$ denotes the conditional probability mass function of term $w_{i}$ in class $c_{j}$. The Bayes discriminant function (1) therefore becomes

$$
\begin{aligned}
f(d)= & \sum_{i: w_{i}(d)=1} \log \frac{P\left(w_{i} \mid c_{1}\right)}{P\left(w_{i} \mid c_{2}\right)}+ \\
& \sum_{i: w_{i}(d)=0} \log \frac{1-P\left(w_{i} \mid c_{1}\right)}{1-P\left(w_{i} \mid c_{2}\right)}+\log \frac{P\left(c_{1}\right)}{P\left(c_{2}\right)},
\end{aligned}
$$

where $P\left(w_{i} \mid c_{j}\right)$ denotes the conditional probability of occurrence of term $w_{i}$ in documents of class $c_{j}$, i.e., $P\left(w_{i} \mid c_{j}\right)=P\left(w_{i}(\cdot)=1 \mid c_{j}\right)$. The log likelihood ratios in (4) are, essentially, term weighting factors that attain large absolute values for terms that are strong discriminators between a pair of classes.

This framework is not limited to binary classification problems. The Bayes discriminant function for a general $K$-class classification task can be written as

$$
g(d)=\arg \max _{j=1, \ldots, K} P\left(c_{j} \mid d\right) .
$$

Given a set of class labels $C=\left\{c_{1}, \ldots, c_{K}\right\}$ and the corresponding (training) set $D_{j}$ of documents representing class $c_{j}$ for each $j \in\{1, \ldots, K\}$, conditional probabilities $P\left(w_{i} \mid c_{j}\right)$ are estimated by

$$
P\left(w_{i} \mid c_{j}\right)=\frac{1+\sum_{d \in D_{j}} w_{i}(d)}{2+\left|D_{j}\right|},
$$

which is the ratio of the number of documents that contain term $w_{i}$ in class $c_{j}$ to the total number of documents in class $c_{j}$. The constants in numerator and 
denominator in (6) are introduced according to Laplace's rule of succession in order to avoid zero-probability terms.

The prior class probabilities $P\left(c_{j}\right)$ are estimated by

$$
P\left(c_{j}\right)=\frac{\left|D_{j}\right|}{\sum_{k=1}^{K}\left|D_{k}\right|} .
$$

Together, equations (3) and (5) 7) comprise the well-known Naive Bayes classifier.

\subsection{Higher Order Data Representation}

The data representation on which we build in the following section was initially used in our prior work 4 for anomaly detection in time series data. In the representation employed herein, a set $D$ of documents is considered as a bipartite graph $G=\left(V_{D} \cup V_{W}, E\right)$. Vertices in $V_{D}$ correspond to documents, while vertices in $V_{W}$ correspond to terms. Two vertices $d \in V_{D}$ and $w \in V_{W}$ are connected by an edge $(d, w) \in E$ iff document $d$ contains the term $w$.

A higher-order path in dataset $D$ is a chain subgraph of $G$. For example, a chain $w_{i}-d_{l}-w_{k}-d_{r}-w_{j}$, which we will denote by $\left(w_{i}, d_{l}, w_{k}, d_{r}, w_{j}\right)$, encodes the fact that document $d_{l} \in D$ contains terms $w_{i}$ and $w_{k}$, while document $d_{r} \in D$ contains terms $w_{k}$ and $w_{j}$. The order of a path is determined by the number of document vertices the path spans. Thus, path $\left(w_{i}, d_{l}, w_{k}, d_{r}, w_{j}\right)$ captures a second-order co-occurrence between terms $w_{i}$ and $w_{j}$, realized through term $w_{k}$ shared by distinct documents $d_{l}$ and $d_{r}$.

Higher-order paths simultaneously capture term co-occurrences within documents as well as term sharing patterns across documents, and in doing so provide a much richer data representation than the traditional feature vector form. As we will see in Sect. 5, the richness of representation becomes crucial when the small size of a training dataset prohibits traditional methods from accurately estimating model parameters.

\section{Approach}

In this section we present two novel methods of leveraging higher-order dependencies between features for supervised machine learning. In Sect. 4.1, we build on the Naive Bayes classifier by introducing a higher-order classifier termed Higher Order Naive Bayes. Our approach, however, can be generalized beyond the probabilistic machine learning methods. In Sect. 4.2, we introduce a datadriven space transformation that allows any learner that operates in vector spaces to leverage higher-order dependencies in data.

\subsection{Higher Order Naive Bayes}

Higher-order paths as defined in Sect. 3.2 allow us to extract rich relational information between features in a dataset. We incorporate this information into 
a Bayesian learning framework by modifying the parameter estimation equations (6) and (7) to depend on the counts of higher-order paths as follows.

Let $\varphi\left(w_{i}, D\right)$ denote the number of higher-order paths that contain term $w_{i}$ given the dataset $D$, and let $\Phi(D)$ denote the total number of higher-order paths in $D$. The parameter estimation equations of the proposed Higher Order Naive Bayes classifier are:

$$
\hat{P}\left(w_{i} \mid c_{j}\right)=\frac{1+\varphi\left(w_{i}, D_{j}\right)}{2+\Phi\left(D_{j}\right)},
$$

and

$$
\hat{P}\left(c_{j}\right)=\frac{\Phi\left(D_{j}\right)}{\sum_{k=1}^{K} \Phi\left(D_{k}\right)} .
$$

\subsection{Leveraging Higher Order Dependencies by a Vector Space-Based Classifier}

In this section, we present a novel data transformation that allows any classifier operating in vector spaces to take advantage of higher-order dependencies between features. We describe our approach for the case of binary classification. This, however, does not limit the applicability of the proposed approach, because numerous methods for multi-class classification based on binary classifiers have been proposed (see [16] for an overview). The proposed data transformation proceeds as follows.

Given two sets $D_{j}$ and $D_{k}$ of (training) documents from classes $c_{j}$ and $c_{k}$, resp., the class conditional term probabilities (8) are computed. Let us denote the corresponding conditional log likelihood ratios as

$$
\phi_{i}^{(1)}=\log \frac{\hat{P}\left(w_{i} \mid c_{j}\right)}{\hat{P}\left(w_{i} \mid c_{k}\right)},
$$

and

$$
\phi_{i}^{(0)}=\log \frac{1-\hat{P}\left(w_{i} \mid c_{j}\right)}{1-\hat{P}\left(w_{i} \mid c_{k}\right)} .
$$

Each binary document vector $d=\left(d_{1}, \ldots, d_{n}\right), d \in D_{j} \cup D_{k}$, is then transformed into a real vector $\hat{d}=\left(\hat{d}_{1}, \hat{d}_{2}, \ldots, \hat{d}_{n}\right)$, where

$$
\hat{d}_{i}=\left\{\begin{array}{l}
\frac{\phi_{i}^{(1)}}{\sqrt{\left|\phi_{i}^{(1)}\right|}}, \text { if } d_{i}=1, \phi_{i}^{(1)} \neq 0 \\
\frac{\phi_{i}^{(0)}}{\sqrt{\left|\phi_{i}^{(0)}\right|}}, \text { if } d_{i}=0, \phi_{i}^{(0)} \neq 0 \\
0, \text { otherwise. }
\end{array}\right.
$$

Finally, the resulting dataset $\hat{D}_{j} \cup \hat{D}_{k}$ is used as input for training a binary classifier for classes $c_{j}$ and $c_{k}$. 
Data transformation (12) assigns weights that are high in absolute values for highly discriminative terms present in a document. The normalizing factors 2 in (12) moderate the spread of values of each feature in order to allow less discriminative terms to retain a certain level of influence over the classification. This level of influence depends on the discriminative power of a term as measured by (10) and (11). An illustration of the effect of the normalizing factors can be found in Sect. 6.

\section{$5 \quad$ Experimental Results}

Experimental evaluation was carried out on six widely-used text corpora. Four of these datasets were RELIGION, SCIENCE, POLITICS and COMP subsets of the 20 News Groups (20NG) [17] benchmark data. These particular subsets were selected to allow us to draw comparisons of our results with the related work [6], which will be discussed later in this section. Our preprocessing procedures closely followed those in 6]. First, all cross-postings in the $20 \mathrm{NG}$ data were removed. Then, for each dataset we performed stop word removal, stemming and removal of all terms that occurred in fewer than three documents in the dataset. The remaining terms were ranked by Information Gain. The top 2000 terms were selected. Finally, 500 documents were sampled at random from each class to comprise the $20 \mathrm{NG}$ datasets used in our experiments.

The other two datasets, Citeseer and Cora, are collections of scholarly research articles preprocessed by [18. The Citeseer dataset contained 3312 documents with a vocabulary of 3703 terms. The Cora dataset comprised of 2708 documents with a vocabulary of 1433 terms. A summary description of all six datasets is provided in Table 1.

Naive Bayes (NB) was used as the baseline for evaluation of the proposed Higher Order Naive Bayes (HONB) classifier. Support Vector Machine (SVM) [19] was chosen as the base classifier for evaluation of the data transformation

Table 1. Six datasets used in the experiments

\begin{tabular}{|c|l|}
\hline Dataset & Classes \\
\hline RELIGION (3) & alt.atheism, soc.religion.christian, talk.religion.misc \\
\hline SCIENCE (4) & sci.crypt, sci.electronics, sci.med, sci.space \\
\hline POLITICS (3) & talk.politics.guns, talk.politics.mideast, talk.politics.misc \\
\hline COMP (5) & $\begin{array}{l}\text { comp.graphics, comp.os.ms-windows.misc, } \\
\text { comp.sys.ibm.pc.hardware, comp.sys.mac-hardware, comp.windows.x }\end{array}$ \\
\hline Citeseer (6) & AI, Agents, DB, HCI, IR, ML \\
\hline Cora (6) & $\begin{array}{l}\text { Case_Based, Genetic_Algorithms, Neural_Networks, Probabilis- } \\
\text { tic_Methods, Reinforcement_Learning, Theory }\end{array}$ \\
\hline
\end{tabular}

${ }^{2}$ It is possible to omit the normalizing factors in (12). However, we have found experimentally that the normalized transformation, on average, yields slightly higher classification accuracies. 
proposed in Sect. 4.2. SVM with the linear kernel has been shown [20] to perform well on text classification problems. The linear kernel allows us to observe the direct impact of leveraging higher-order dependencies, without any additional data transformations as performed implicitly by other kernel functions. Multiclass problems were addressed using the "one-against-one" classification scheme 21. Under this scheme, a binary SVM classifier is constructed for every pair of classes. A data instance is then classified by each binary classifier and the final classification is determined by the majority vote over the assigned class labels. We refer to a SVM classifier constructed on the transformed data as Higher Order SVM (HOSVM).

Experiments described in this section were done using second-order paths for HONB and HOSVM. A $O\left(n^{2}(m+n)\right)$ algorithm for obtaining the counts of second-order paths in a dataset with $m$ instances in $n$ dimensions can be found in [22. We have conducted additional experiments using third-order paths, but the results did not differ significantly.

Figure 2 shows mean classification accuracies obtained by varying training set size from $5 \%$ up to $60 \%$. For each training set size, eight trials were performed. On each trial, a set of documents were randomly sampled from each class for training, while the rest were used for testing. On every trial, all terms that did not appear in any of the training documents were disregarded. The classifiers were then trained in the corresponding subspace of the original term space.

As can be seen from Figs. 2(a) 2(d), HONB and HOSVM 3 consistently outperformed first-order classifiers $\mathrm{NB}$ and $\mathrm{SVM}^{3}$, resp., on the RELIGION, SCIENCE, POLITICS and COMP datasets. All of these accuracy improvements were statistically significant at the $5 \%$ level for $(\mathrm{HONB}, \mathrm{NB})$ pair of classifiers and in $83 \%$ of the cases for the (HOSVM, SVM) pair.

A slightly different pattern of performance can be observed on the Citeseer (Fig. 2(e) and Cora (Fig. 2(f) datasets. While classification accuracy of each method monotonically increased with increasing training set size, HONB drastically outperformed NB when training sets were small, reaching close to accuracy levels of SVM-based methods. HOSVM performed 1.5-2\% better than SVM 66\% of the time, but never worse overall. These results are especially encouraging in that they suggest that higher-order methods were able to construct robust models even when training data was particularly scarce. We speculate that changes in the distribution of highly-discriminative terms across classes as a result of increasing training set size allowed NB to outperform HONB on the Citeseer and Cora datasets once the amount of training data reached a certain point. We are currently investigating this hypothesis and intend to report our findings as part of our future work.

Consistent and statistically significant accuracy improvements attained by higher-order classifiers on small training sets led us to explore this aspect

\footnotetext{
${ }^{3}$ When selecting the value of the soft margin cost parameter $C$ for SVM, we considered the set $\left\{10^{-4}, 10^{-3}, \ldots, 10^{4}\right\}$ of possible values. On every trial, we picked the smallest value of $C$ which resulted in the highest accuracy obtained on the training set.
} 


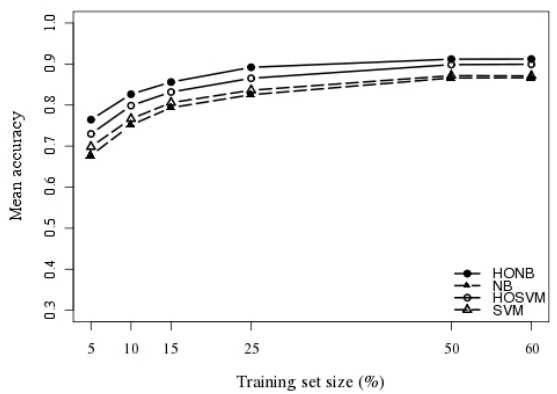

(a) RELIGION

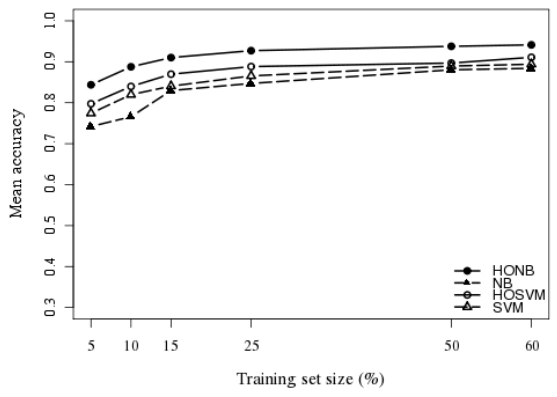

(c) POLITICS

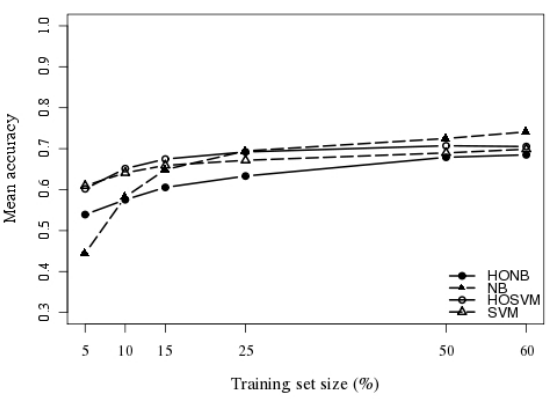

(e) Citeseer

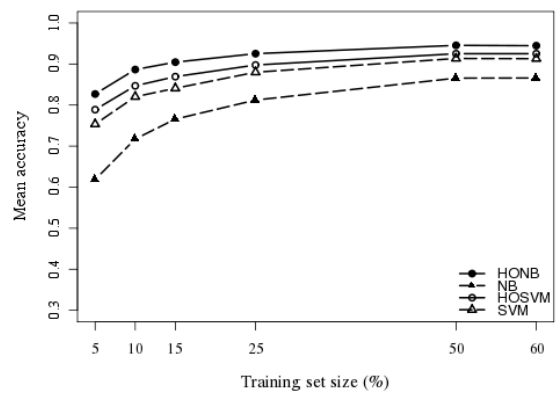

(b) SCIENCE

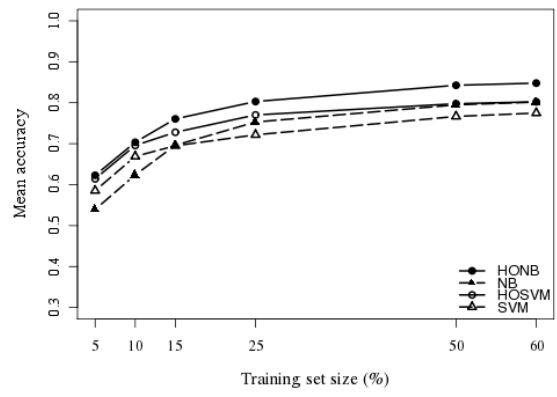

(d) COMP

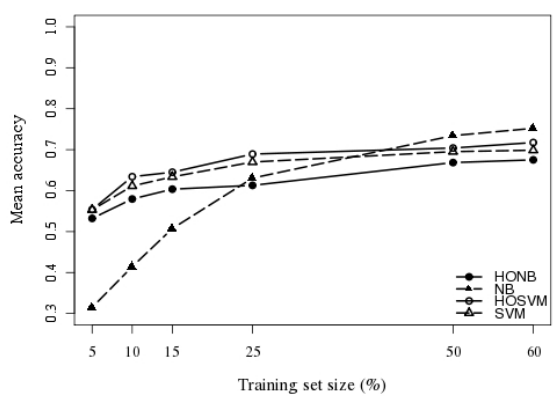

(f) Cora

Fig. 2. Scalability across training set size

further. In order to simulate a real-world scenario where only a few labeled data instances are available, and to illustrate accuracy improvements in a setting comparable with the related work [6], we focused our attention on $5 \%$ training samples. In case of the $20 \mathrm{NG}$ datasets, for instance, this corresponded to training on 25 documents per class and testing on the other 475 documents per 
Table 2. Mean classification accuracies

\begin{tabular}{|c|c|c|c|c||c|c|c|c|}
\hline & \multicolumn{2}{|c|}{ NB } & \multicolumn{2}{c||}{ HONB } & \multicolumn{2}{c|}{ SVM } & \multicolumn{2}{c|}{ HOSVM } \\
\hline Dataset & Acc. & St. dev. & Acc. & St. dev. & Acc. & St. dev. & Acc. & St. dev. \\
\hline RELIGION (3) & 0.66 & 0.051 & $\mathbf{0 . 7 4 1}$ & 0.03 & 0.699 & 0.022 & $\mathbf{0 . 7 2 3}$ & 0.023 \\
\hline SCIENCE (4) & 0.632 & 0.071 & $\mathbf{0 . 8 3 3}$ & 0.043 & 0.751 & 0.029 & $\mathbf{0 . 7 9 2}$ & 0.039 \\
\hline POLITICS (3) & 0.73 & 0.042 & $\mathbf{0 . 8 3 2}$ & 0.01 & 0.763 & 0.03 & $\mathbf{0 . 7 9 3}$ & 0.047 \\
\hline COMP (5) & 0.539 & 0.051 & $\mathbf{0 . 6 2 3}$ & 0.036 & 0.585 & 0.022 & $\mathbf{0 . 6 1 4}$ & 0.031 \\
\hline Citeseer (6) & 0.444 & 0.025 & $\mathbf{0 . 5 3 9}$ & 0.015 & $\mathbf{0 . 6 0 9}$ & 0.01 & 0.602 & 0.01 \\
\hline Cora (6) & 0.315 & 0.006 & $\mathbf{0 . 5 3 2}$ & 0.021 & 0.553 & 0.021 & $\mathbf{0 . 5 5 4}$ & 0.026 \\
\hline
\end{tabular}

class. Classification accuracies averaged over eight trials are reported in Table 2 . Highest accuracies for pairs (NB, HONB) and (SVM, HOSVM) of classifiers are highlighted in bold. The corresponding standard deviations are also reported in Table 2,

The obtained results indicate that leveraging higher-order dependencies in the RELIGION, SCIENCE, POLITICS and COMP datasets lead to significant improvements in classification accuracies of both Bayesian and SVM-based approaches. The improvements of HONB over NB and of HOSVM over SVM on those datasets are statistically significant at the $5 \%$ level. The only exception is the (HOSVM, SVM) pair on the POLITICS dataset. Although the difference in SVM and HOSVM accuracies on the POLITICS dataset was significant at level $\alpha=0.158$, HOSVM outperformed SVM on seven out of eight trials on that data by an average of $3 \%$.

HONB performed particularly well on the 20NG data, outperforming NB by $11.7 \%$ and SVM by $5.8 \%$ on average. HONB also outperformed HOSVM by an average of $2.7 \%$, although the differences in accuracy between the two classifiers were not statistically significant at the $5 \%$ level. HOSVM consistently outperformed SVM by an average of $3.1 \%$.

On the Citeseer dataset, HONB outperformed NB by about $9 \%$ (significant at the $5 \%$ level), while HOSVM and SVM performed at the same level. On the Cora dataset, NB's average classification accuracy was $31.5 \%$, which is the lowest accuracy across all datasets and classifiers considered in this work. Such low accuracy and almost zero standard deviation (Table 2) resulted from NB assigning all test documents to the majority class Neural_Networks. By exploiting valuable higher-order dependencies, HONB attained approximately $53 \%$ average accuracy, which is not significantly different at the $5 \%$ level from the roughly $55 \%$ accuracy of SVM and HOSVM.

In another set of experiments, we expanded our reach to compare the performance of higher-order classifiers to other approaches that also attempt to achieve better generalization performance on sparse input data. These related research efforts use clustering as a dimensionality reduction technique. In general they cluster words into groups and these groups are used as features in text classification. The authors of [6] observed that one advantage of using word clusters can be seen when word statistics (i.e., NB parameter estimates) are relatively hard to estimate. In order to demonstrate this, they conducted experiments with 
Table 3. Mean classification accuracies reported by [

\begin{tabular}{|c|c|c|}
\hline Dataset & mNB & NBWC \\
\hline RELIGION (3) & 0.525 & 0.553 \\
\hline SCIENCE (4) & 0.65 & 0.725 \\
\hline POLITICS (3) & 0.62 & 0.67 \\
\hline COMP (5) & 0.473 & 0.508 \\
\hline
\end{tabular}

Table 4. Mean classification accuracies of a pure higher-order classifier

\begin{tabular}{|c|c|c|}
\hline Dataset & HONB & pure HONB \\
\hline RELIGION (3) & 0.741 & 0.745 \\
\hline SCIENCE (4) & 0.833 & 0.842 \\
\hline POLITICS (3) & 0.832 & 0.836 \\
\hline COMP (5) & 0.623 & 0.649 \\
\hline
\end{tabular}

small numbers of labeled training documents from the subsets listed in Table 1 of the $20 \mathrm{NG}$ data. To provide a more objective comparison, we focused on the relative improvement over respective base models. In [6] the authors used multinomial NB (mNB) as their base model and improved on the performance of this model using word clusters as features instead of just words. The latter algorithm is indicated as NBWC in Table 3. Similarly, we used binomial NB as our base model and improved on the performance of this model using higher-order paths instead of individual documents to estimate parameters.

Tables 2 and 3 show that compared to NBWC, HONB achieved much better performance than the corresponding base model for all datasets $(4.7 \%$ average difference between $\mathrm{NBWC}$ and $\mathrm{mNB}$ versus $11.7 \%$ average difference between HONB and NB). Additionally, our results are statistically significant.

In order to further verify the value of leveraging higher-order dependencies within the data, additional experiments were conducted. In the first experiment, when estimating the conditional probabilities (8) and (9), we used only the "pure" second-order paths, i.e., paths that involved terms which did not cooccur together in any single document of the training set. The results of these experiments are reported in Table 4 comparing performance of HONB with that of the pure HONB. The differences in performance of these classifiers were minimal and not statistically significant.

In the second experiment, prior to training an SVM classifier with the linear kernel, a data transformation analogous to (12) was performed using the firstorder conditional term probabilities (6) instead of higher-order probabilities (8). The resulting approach is referred to as NBSVM. Mean classification accuracies attained by $\mathrm{NBSVM}^{3}$ are shown in Table [5. Comparison of Tables 2 and 5 makes it clear that NBSVM performed worse than both SVM and HOSVM. These results indicate that taking advantage of higher-order dependencies was indeed crucial for achieving the performance improvements attained by HONB and HOSVM. 
Table 5. Mean classification accuracies of NBSVM

\begin{tabular}{|c|c|c|}
\hline Dataset & NBSVM & HOSVM \\
\hline RELIGION (3) & 0.678 & 0.723 \\
\hline SCIENCE (4) & 0.745 & 0.792 \\
\hline POLITICS (3) & 0.759 & 0.793 \\
\hline COMP (5) & 0.576 & 0.614 \\
\hline
\end{tabular}

We have also conducted experiments with the Radial Basis Function (RBF) kernel for the HOSVM and SVM classifiers. The results were consistent with the findings of [20]. Namely, there were no significant differences between classification accuracies attained with the linear kernel and those attained with the RBF kernel.

In summary, higher-order approaches HONB and HOSVM outperformed their first-order counterparts. Consistent performance improvements were observed on the 20NG data across a wide range of training set sizes. On the Citeseer and Cora datasets, HONB outperformed NB when training set sizes were below $10 \%$ and $25 \%$, respectively. Under the conditions of small (5\%) training samples from the Citeseer dataset, HOSVM performed the same as SVM, which outperformed HONB by $7 \%$. HONB, SVM and HOSVM produced very similar results on the Cora dataset. It is interesting to note that while HOSVM scaled better across datasets, HONB did not require any parameter tuning and performed exceptionally well on the $20 \mathrm{NG}$ data.

\section{Discussion}

In order to gain a deeper understanding of the effect of using higher-order paths for estimation of conditional term probabilities (8), let us consider Fig. 3 generated based on one of the 5\% (25 documents per class) training samples from the RELIGION dataset using two of its classes, "alt.atheism" and "soc.religion.christian". For every term $w_{i}$, Fig. 3(a) presents a plot of the conditional log probability ratio (10) obtained from higher-order probabilities (8) (horizontal axis) versus the log ratio obtained from first-order probabilities (6) (vertical axis). Notice the differences in scales of values on the axes of Fig. 3(a). $[-20,20]$ for higher-order log ratios versus $[-4,4]$ for first-order log ratios. Additionally, three distinct groups of terms appeared as a result of using higher-order paths for estimating the model parameters. We found that terms that fell into the right (left) most group are highly-discriminative terms that appeared in documents of only one of the classes in the training set. Figure 3(a) reveals that due to drastic difference in scales of values of higher- and first-order log ratios, highly-discriminative terms exert much stronger influence on classification in HONB than in NB. In other words, highly-discriminative terms contribute more heavily to the Bayesian discriminant function (4) under HONB than under NB.

Similarly, Fig. 3(b) shows a plot of the conditional log probability ratios (11) of non-occurrence of a term in a HONB model versus a NB model. An important feature in this figure is the one order of magnitude difference in values 


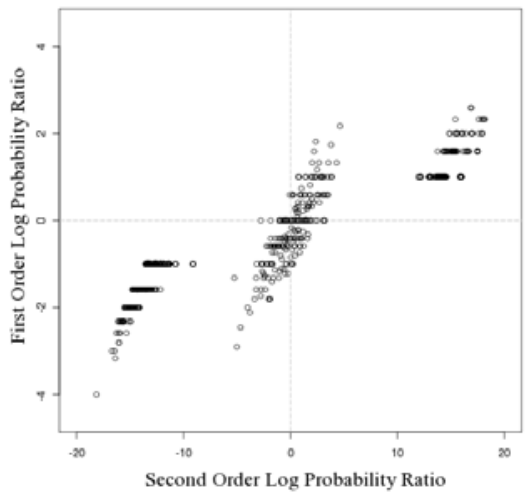

(a)

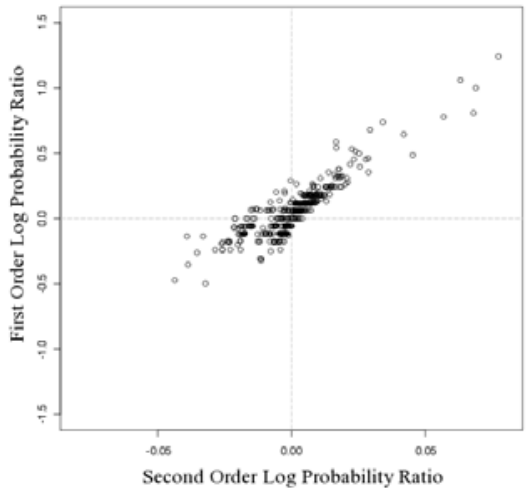

(b)

Fig. 3. Conditional log probability ratios obtained from higher-order probabilities (8) (horizontal axes) versus the log ratios obtained from first-order probabilities (6) (vertical axes) on "alt.atheism" and "soc.religion.christian" classes of one of the $5 \%$ (25 documents per class) training samples from the RELIGION dataset

on the axes: $[-0.1,0.1]$ for higher-order log ratios on the horizontal axis versus $[-1.5,1.5]$ for first-order log ratios on the vertical axis. Together, Figs. 3(a) and 3(b) indicate that while both NB and HONB take into account presence of terms as well as their absence, HONB tends to place more emphasis on the presence of terms in a document being classified.

As was noted in Sect. 4.2, the normalizing factors in (12) were introduced in order to allow terms that may appear in multiple classes, but are still good discriminators as measured by the log likelihood ratios (10) and (11), to have a non-negligible impact during document classification by HOSVM. The effect of these normalizing factors can be seen by comparing Figs. 3(a) and 3(b) with Figs. 4(a) and 4(b).

On the vertical axes in Figs. 4(a) and 4(b) are plotted the same first-order conditional log probability ratios as in Figs. $3(\mathrm{a})$ and 3(b) respectively. Plotted on the horizontal axes of Figs. 4(a) and 4(b) are the higher-order conditional log probability ratios shown on the horizontal axes of Figs. 3(a) and 3(b), respectively, and normalized as in (12). Note the change in scales of the horizontal axes once normalization has been applied: $[-20,20]$ before normalization (Fig. $3(\mathrm{a})$ versus $[-4,4]$ after, and $[-0.1,0.1]$ before normalization (Fig. 3(b) versus $[-0.3,0.3]$ after. It is this change in scales coupled with the increased spread of the middle group of terms along the horizontal axis that allowed good discriminator terms from the middle group in Fig. 3(a) to increase their relative influence during document classification by HOSVM.

Although it is trivial to identify strongly discriminative features in a given training set, the question remains of how to weight those features for pattern classification. Methods proposed in this work address this question by leveraging higher-order dependencies between features. 


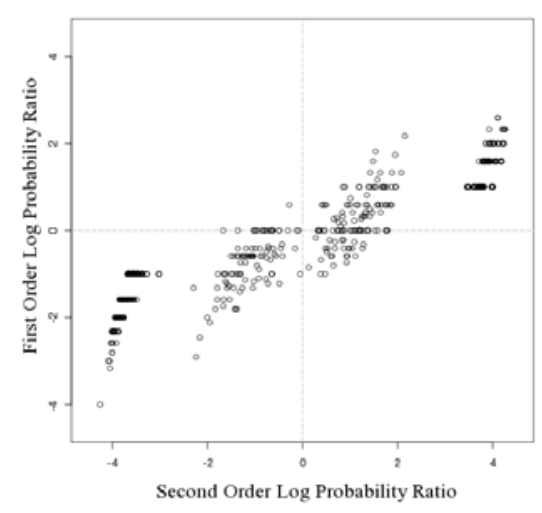

(a)

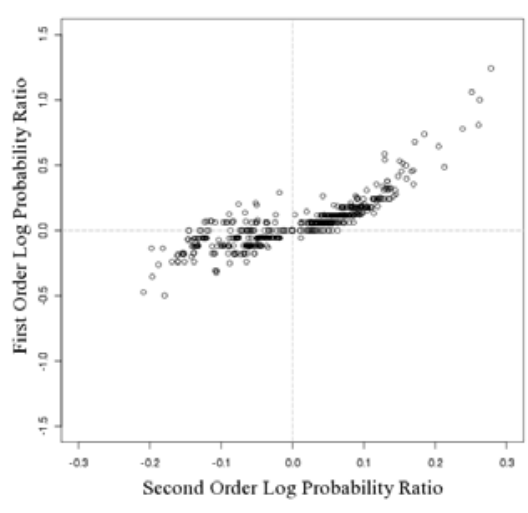

(b)

Fig. 4. Conditional log probability ratios obtained from higher-order probabilities (8) and normalized as in (12) for use by HOSVM (horizontal axes) versus the log ratios obtained from first-order probabilities (6) (vertical axes) on the same dataset as in Fig. 3

\section{Conclusions and Future Work}

In prior work [5], we gave a mathematical proof supported by empirical results of the dependence of LSI on higher-order paths. In this work, a general approach to leveraging higher-order dependencies for supervised learning was developed. We presented a novel classification method termed Higher Order Naive Bayes (HONB). We further generalized our framework by developing a new data transformation that allows any classifier operating in vector spaces to take advantage of the rich relational information captured by higher-order paths.

Higher-order paths allow a classifier to operate on a much richer data representation than the conventional feature vector form. This is especially important when working with small and sparse training sets where accurate parameter estimation of traditional (first-order) models becomes very challenging [6]. Experimental results affirmed the value of leveraging higher-order paths, resulting in significant improvements in classification accuracies on benchmark text corpora across a wide range of training set sizes. In addition, we compared our results with those reported in [6], where term clusters were used (instead of terms) as features for classification by Naive Bayes (NBWC). Our experiments demonstrated that HONB consistently achieved better performance over the baseline Naive Bayes (NB) classifier than did NBWC.

In ongoing efforts, we are developing methods for leveraging higher-order dependencies in various domains including nuclear detection, as well as in large datasets. We are also investigating extensions of the proposed framework to a number of other supervised learning approaches, and to unsupervised machine learning. 


\section{Acknowledgments}

The authors wish to thank Rutgers University, the National Science Foundation and the Department of Homeland Security. This material is based upon work partially supported by the National Science Foundation under Grant Numbers 0703698 and 0712139 as well as by the Department of Homeland Security Science \& Technology Directorate. Any opinions, findings, and conclusions or recommendations expressed in this material are those of the authors and do not necessarily reflect the views of the National Science Foundation, the Department of Homeland Security or Rutgers University.

The authors also wish to thank several colleagues at DIMACS, Rutgers University, including Dr. Ilya B. Muchnik for valuable discussions regarding the higher-order path counting algorithm, Dr. Alexey V. Nefyodov for helpful suggestions on the presentation of this work and Shenzhi Li for her many insightful comments during the development of the Higher Order Learning framework.

We are also grateful for the help of other co-workers, family members and friends. Co-author W. M. Pottenger also gratefully acknowledges the continuing help of his Lord and Savior, Yeshua the Messiah (Jesus the Christ) in his life and work.

\section{References}

1. Chakrabarti, S., Dom, B., Indyk, P.: Enhanced hypertext categorization using hyperlinks. SIGMOD Rec. 27(2), 307-318 (1998)

2. Neville, J., Jensen, D.: Iterative classification in relational data. In: Proc. AAAI, pp. 13-20. AAAI Press, Menlo Park (2000)

3. Taskar, B., Segal, E., Koller, D.: Probabilistic classification and clustering in relational data. In: Proceedings of the Seventeenth International Joint Conference on Artificial Intelligence, pp. 870-878 (2001)

4. Ganiz, M.C., Kanitkar, S., Chuah, M.C., Pottenger, W.M.: Detection of interdomain routing anomalies based on higher-order path analysis. In: ICDM 2006: Proceedings of the Sixth International Conference on Data Mining, pp. 874-879. IEEE Computer Society, Los Alamitos (2006)

5. Kontostathis, A., Pottenger, W.M.: A framework for understanding latent semantic indexing (LSI) performance. Inf. Process. Manage. 42(1), 56-73 (2006)

6. Slonim, N., Tishby, N.: The power of word clusters for text classification. In: 23rd European Colloquium on Information Retrieval Research (2001)

7. Getoor, L., Diehl, C.P.: Link mining: a survey. SIGKDD Explor. Newsl. 7(2), 3-12 (2005)

8. Lu, Q., Getoor, L.: Link-based classification. In: Fawcett, T., Mishra, N. (eds.) ICML, pp. 496-503. AAAI Press, Menlo Park (2003)

9. Neville, J., Jensen, D.: Dependency networks for relational data. In: Fourth IEEE International Conference on Data Mining, 2004. ICDM 2004, pp. 170-177 (2004)

10. Deerwester, S., Dumais, S.T., Furnas, G.W., Landauer, T.K., Harshman, R.: Indexing by latent semantic analysis. Journal of the American Society for Information Science 41, 391-407 (1990) 
11. Li, S., Wu, T., Pottenger, W.M.: Distributed higher order association rule mining using information extracted from textual data. SIGKDD Explor. Newsl. 7(1), 26-35 (2005)

12. Edmonds, P.: Choosing the word most typical in context using a lexical cooccurrence network. In: Proceedings of the 35th Annual Meeting of the Association for Computational Linguistics, pp. 507-509 (1997)

13. Zhang, X., Berry, M.W., Raghavan, P.: Level search schemes for information filtering and retrieval. Inf. Process. Manage. 37(2), 313-334 (2001)

14. Schütze, H.: Automatic word sense discrimination. Comput. Linguist. 24(1), 97 123 (1998)

15. Xu, J., Croft, W.B.: Corpus-based stemming using cooccurrence of word variants. ACM Trans. Inf. Syst. 16(1), 61-81 (1998)

16. Scholkopf, B., Smola, A.J.: Learning with Kernels: Support Vector Machines, Regularization, Optimization, and Beyond. MIT Press, Cambridge (2001)

17. Lang, K.: Newsweeder: Learning to filter netnews. In: Proceedings of the Twelfth International Conference on Machine Learning, pp. 331-339 (1995)

18. Sen, P., Getoor, L.: Link-based classification. Technical Report CS-TR-4858, University of Maryland (February 2007)

19. Vapnik, V.: Statistical Learning Theory. John Wiley, Chichester (1998)

20. Joachims, T.: Learning to Classify Text Using Support Vector Machines: Methods, Theory and Algorithms. Kluwer Academic Publishers, Norwell (2002)

21. Kreßel, U.H.G.: Pairwise classification and support vector machines. In: Advances in kernel methods: support vector learning, pp. 255-268. MIT Press, Cambridge (1999)

22. Ganiz, M.C., Lytkin, N.I., Pottenger, W.M.: Leveraging higher order dependencies between features for text classification. Technical Report 2009-16, DIMACS, Rutgers University (June 2009) 